\title{
Microscopy in the Study of the Eye Disease Glaucoma
}

\author{
Cheryl R. Hann ${ }^{1}$ and Michael P. Fautsch ${ }^{1}$ \\ ${ }^{1 .}$ Department of Ophthalmology, Mayo Clinic, Rochester, MN
}

Glaucoma is the leading cause of irreversible blindness affecting millions of people worldwide. Elevated intraocular pressure is the most prevalent and only treatable risk factor for the disease. In the normal eye, intraocular pressure is determined by the balance between the amount of aqueous humor produced by the ciliary body non-pigmented epithelial cells and the amount that is removed from the front of the eye through an outflow pathway. The main outflow pathway consists of a filter-like tissue called the trabecular meshwork where aqueous humor flows through and drains into a $360^{\circ}$ collection vessel at the junction of the cornea and sclera called Schlemm's canal. From Schlemm's canal, aqueous humor passes through collector channels that drain into the episcleral venous system (Fig 1). In glaucoma, elevated intraocular pressure occurs by a dysfunction in this outflow pathway. Microscopy has played a significant role in defining the proximal and distal portions of the outflow pathway in normal and in glaucoma patients. The purpose of this paper is to define how we use multiple imaging modalities to trace outflow in the eye, identify composition of eye tissues and examine 3-dimensional structure in normal and glaucoma tissues.

Research in our lab has utilized fluorescently-labeled microspheres to map fluid movement through the trabecular outflow pathway. Using this technique, we identified preferential pathways within the trabecular meshwork adjacent to collector channels located on the distal side of Schlemm's canal (Fig 1). This significant finding suggested that the trabecular outflow pathway may be working as a unit combining both proximal and distal regions to actively move fluid through the pathway. To evaluate the entire pathway, we utilized 3-dimensional micro-computed tomography (Fig. 2) and compared normal and glaucoma eyes at low and high perfusion pressures. In normal eyes, Schlemm's canal volume, collector channel orifice area and collector channel diameter were increased compared to eyes with glaucoma at like pressures. Additionally, glaucoma eyes at elevated pressure had a 3.7 fold increase in occluded collector channels when compared to normal eyes at like pressure elevation, due to the narrowed volume and tissue from the proximal part of Schlemm's canal blocking the collector channel opening on the distal side. We are also using 2-photon and single harmonic generation with confocal imaging to investigate the extracellular attachments between the trabecular meshwork and Schlemm's canal. These recent studies show a decrease in elastin near the base of Schlemm's canal glaucoma eyes (Fig 3) which may affect the mechano-sensitive environment and disrupt outflow. In conclusion, we are utilizing multiple imaging modalities to answer questions regarding fluid flow patterns, local and global relationships within the eye, and morphological changes that occur in glaucoma.

\section{References:}

[1] CR Hann and MP Fautsch, Invest Ophthalmol Vis Sci (2009) 1692.

[2] CR Hann et al., Exp Eye Res (2010) 104.

[3] CR Hann et al., Invest Ophthalmol Vis Sci (2014) 5834. 

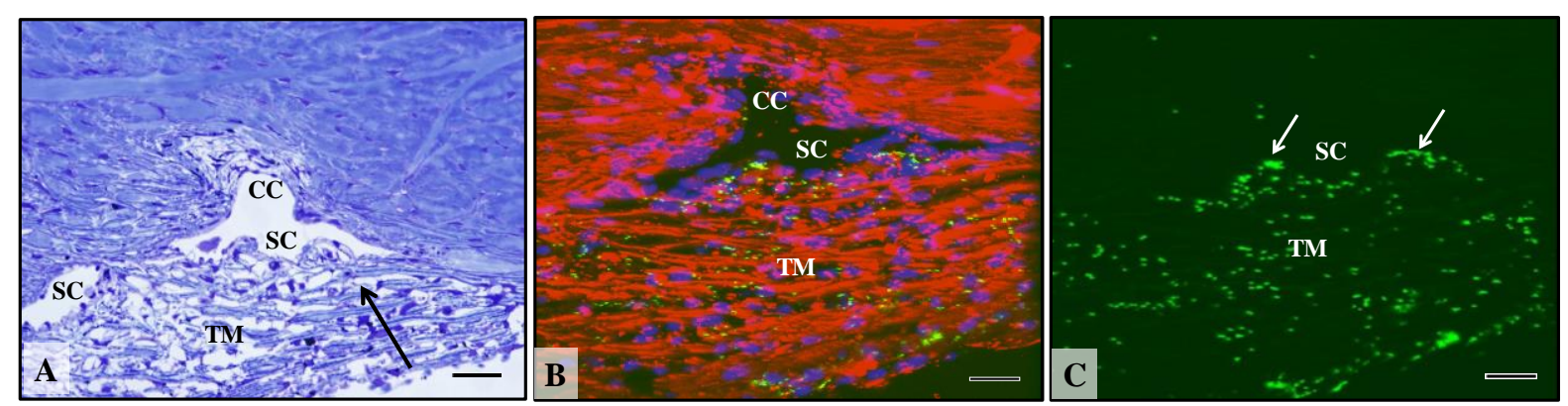

Figure 1. (A) Toluidine blue stained section of a normal outflow pathway showing trabecular meshwork (TM), Schlemm's canal (SC), and collector channel (CC). Black arrow indicates direction of fluid flow. (B) Confocal images showing trabecular meshwork (TM), Schlemm's canal (SC), and collector channel (CC) following perfusion with fluorescently-labeled microspheres. (C) Accumulation of fluorescent microspheres in expanded regions (white arrows) adjacent to CC orifice. Scale bar $=20 \mu \mathrm{m}$.

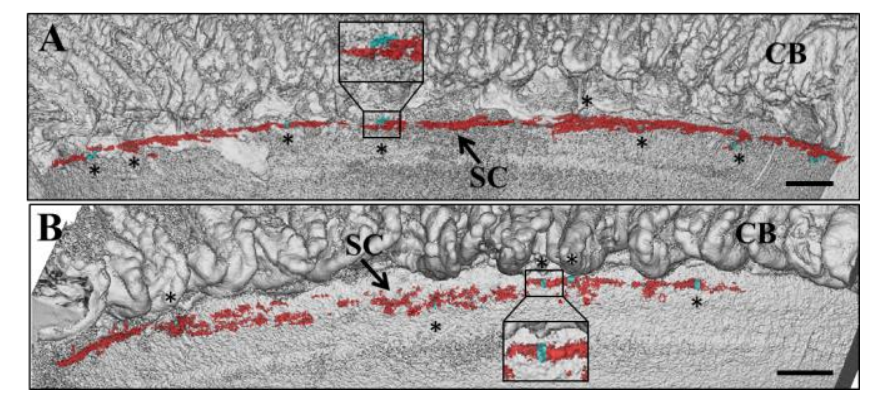

Figure 2. (A) 3-dimensional micro-computed tomography of a normal eye perfused at $20 \mathrm{mmHg}$. (B) 3-dimensional micro-computed tomography of a glaucoma eye perfused at $20 \mathrm{mmHg}$. Schlemm's canal (SC) red and collector channels (CCs) aqua. Glaucoma volumes show reduced SC area and volume (red). Ciliary body (CB). Scale bar $=1000 \mu \mathrm{m}$.
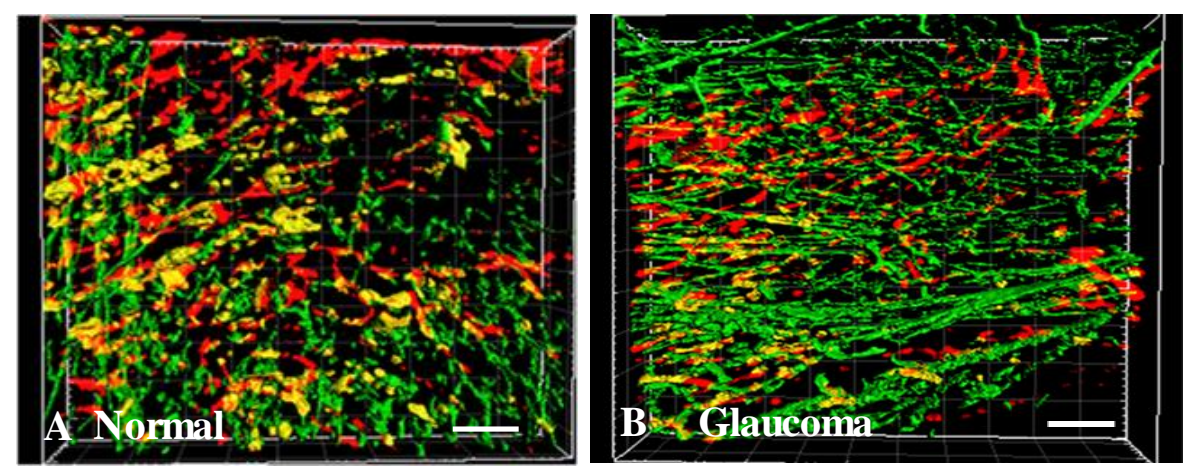

Figure 3. (A) 2-Photon normal eye volume rendered in IMARIS. (B) Glaucoma eye volume rendered in IMARIS. Elastin, green; elastin within $1 \mu \mathrm{m}$ of cell membrane, yellow; and cell membrane, red. Analysis in IMARIS found glaucoma eyes had less elastin within $1 \mu \mathrm{m}$ of cell membrane indicating possible changes in the mechano-sensitive environment in the trabecular meshwork. Scale bar $=15 \mu \mathrm{m}$. 